\title{
Multiplicity Results for Variable-Coefficient Singular Third-Order Differential Equation with a Parameter
}

\author{
Zhibo Cheng $^{1}$ and Yun Xin ${ }^{2}$ \\ ${ }^{1}$ School of Mathematics and Information Science, Henan Polytechnic University, Jiaozuo 454000, China \\ ${ }^{2}$ College of Computer Science and Technology, Henan Polytechnic University, Jiaozuo 454000, China \\ Correspondence should be addressed to Zhibo Cheng; czb_1982@126.com
}

Received 24 March 2014; Accepted 9 June 2014; Published 19 June 2014

Academic Editor: Narcisa C. Apreutesei

Copyright (c) 2014 Z. Cheng and Y. Xin. This is an open access article distributed under the Creative Commons Attribution License, which permits unrestricted use, distribution, and reproduction in any medium, provided the original work is properly cited.

\begin{abstract}
We investigate a class of variable coefficients singular third-order differential equation with superlinearity or sublinearity assumptions at infinity for an appropriately chosen parameter. By applications of Green's function and the Krasnoselskii fixed point theorem, sufficient conditions for the existence of positive periodic solutions are established.
\end{abstract}

\section{Introduction}

Generally speaking, differential equations with singularities have been considered from the very beginning of the discipline. The main reason is that singular forces are ubiquitous in applications, the most obvious examples being gravitational and electromagnetic forces. In 1965, Ding [1] discussed the Brillouin electron beam focusing system:

$$
x^{\prime \prime}+a(1+\cos 2 t) x=\frac{1}{x}
$$

and obtained the existence of positive periodic solution for the model if $0<a<1 / 4$.

Ding's work has attracted the attention of many specialists in differential equations. More recently, the method of lower and upper solutions, Poincaré-Birkhoff twist theorem, Mawhin's topological degree theorem, Schauder's fixed point theorem, and Krasnoselskii fixed point theorem in a cone have been employed to investigate the existence of positive periodic solutions of singular second order differential equations (see, e.g., [2-13]). For example, in 2007, Torres [10] studied singular forced semilinear differential equation:

$$
x^{\prime \prime}+a(t) x^{\prime}=f(t, x)+e(t) .
$$

By Schauder's fixed point theorem, the author has shown that the additional assumption of a weak singularity enabled the obtention of new criteria for the existence of periodic solutions. Afterwards, Wang [13] investigated the existence and multiplicity of positive periodic solutions of the singular systems (2) by Krasnoselskii fixed point theorem. The conditions he presented to guarantee the existence of positive periodic solutions are beautiful.

At the beginning, most of work concentrated on secondorder singular differential equations, as in the references we mentioned above. Recently, there have been published some results on third-order singular differential equation (see [1419]). For example, in [14], Chu and Zhou considered the following third-order singular differential equation:

$$
u^{\prime \prime \prime}+\kappa^{3} u=f(t, u), \quad 0 \leq t \leq 2 \pi
$$

with periodic boundary conditions $u^{(i)}(0)=u^{(i)}(2 \pi), i=$ $0,1,2$. Here, $\kappa$ is a positive constant and nonlinearity $f(t, u)$ may be singular at $u=0$. They discussed (3) by transforming it into a first-order equation and a second-order equation. Restricted by Green's function of the second-order differential equation, they obtained existence theorem of periodic solutions for (3) in a small range of $\kappa$, and, to be concrete, $\kappa \in(0,1 / \sqrt{3})$. Afterward, Li [16] investigated the third-order ordinary differential equation:

$$
u^{\prime \prime \prime}(t)=f\left(t, u(t), u^{\prime}(t), u^{\prime \prime}(t)\right), \quad t \in \mathbb{R}
$$


where $f \in C(\mathbb{R} \times(0, \infty) \times \mathbb{R} \times \mathbb{R})$ is $\omega$-periodic in $t$, and $f(t, u, v, w)$ may be singular at $u=0$. By applying a fixed point theorem in cones, the author obtained existence results of positive $\omega$-periodic solutions for (4). Recently, Ren et al. [19] studied the third-order singular nonlinear differential equation:

$$
x^{\prime \prime \prime}(t)+a x^{\prime \prime}(t)+b x^{\prime}(t)+c x(t)=f(t, x(t))+e(t),
$$

where $e(t)$ takes positive values. Using Green's function for third-order differential equation and some fixed-point theorems, that is, Leray-Schauder alternative principle and Schauder's fixed point theorem, they established three new existence results of positive periodic solutions for (5).

In the above papers, the authors investigated singular third-order equations with constant coefficients. However, the study on the singular third-order equation with variable coefficients is relatively infrequent. Motivated by Torres et al. $[10,13,14,16,19]$, in this paper, we consider the singular thirdorder differential equation with variable coefficients:

$$
\begin{aligned}
x^{\prime \prime \prime} & (t)+a(t) x^{\prime \prime}(t)+b(t) x^{\prime}(t)+c(t) x(t) \\
& =\mu g(t) f(x(t))+\mu e(t),
\end{aligned}
$$

where $\mu>0$ is a positive parameter, and $e(t)$ may take positive value or negative value. $a, b, c \in C\left(\mathbb{R}, \mathbb{R}^{+}\right)$are $\omega$ periodic functions; $g(t), e(t)$ are $\omega$-periodic continuous scalar functions in $t \in \mathbb{R}$. The nonlinear term $f$ of (6) can be with a singularity at origin; that is,

$$
\lim _{x \rightarrow 0^{+}} f(x)=+\infty, \quad\left(\text { or } \lim _{x \rightarrow 0^{+}} f(x)=-\infty\right) .
$$

It is said that (6) is of repulsive type (resp., attractive type) if $f(x) \rightarrow+\infty$ (resp., $f(x) \rightarrow-\infty$ ) as $x \rightarrow 0^{+}$.

As far as we know, studies on third-order differential equation with variable coefficients are rather infrequent, especially those focused on the research of singular thirdorder differential equations with variable coefficients. The main difficulty lies in the calculation of Green's function of the third-order differential equation with variable coefficients, being more complicated than in the constant-coefficient case. Therefore, in Section 2, we first study Green's function of the above mentioned third-order differential equation. In Section 3, we define a cone and discuss several properties of the equivalent operator on the cone. In order to simplify the proof in Section 3, we establish a series of lemmas and corollaries to estimate the operator. All the corollaries are the corresponding results for $e(t)$ taking negative values. In Section 4, by employing Green's function and Krasnoselskii fixed point theorem, we state and prove the existence of positive periodic solutions for singular third-order differential equation with superlinearity or sublinearity assumptions at infinity for an appropriately chosen parameter. The result is applicable to the case of a strong singularity as well as the case of a weak singularity. Our results improve and extend the results in $[10,14,19]$.

\section{Green's Function of Third-Order Differential Equation}

Let $X=\{\phi \in C(\mathbb{R}, \mathbb{R}): \phi(t+\omega)=\phi(t)\}$ with the maximum norm $\|\phi\|=\max _{0 \leq t \leq \omega}|\phi(t)|$. Obviously, $X$ is a Banach space. For a given function $e \in L^{1}[0, \omega]$, we denote the essential supremum and infimum by $e^{*}$ and $e_{*}$, if they exist.

Firstly, we consider

$$
\begin{gathered}
x^{\prime \prime \prime}(t)+a(t) x^{\prime \prime}(t)+b(t) x^{\prime}(t)+c(t) x(t)=h(t), \\
x^{(i)}(0)=x^{(i)}(\omega), \quad i=0,1,2,
\end{gathered}
$$

where $h \in C\left(\mathbb{R}, \mathbb{R}^{+}\right)$is an $\omega$-periodic function. Obviously, the calculation of Green's function of (8) is very complicated, so, by analysis of the third-order differential equation (8), we consider only the following two cases.

Case 1. There exist differentiable $\omega$-periodic functions $p$ and $q$ and a positive real constant $\rho$ such that $a(t)=\rho+p(t)$, $b(t)=\rho p(t)+q(t)+p^{\prime}(t)$, and $c(t)=\rho q(t)+q^{\prime}(t)$. Then, (8) is transformed into

$$
\begin{gathered}
y^{\prime}(t)+\rho y(t)=h(t), \\
y(0)=y(\omega), \\
x^{\prime \prime}(t)+p(t) x^{\prime}(t)+q(t) x(t)=y(t), \\
x(0)=x(\omega), \quad x^{\prime}(0)=x^{\prime}(\omega) .
\end{gathered}
$$

Then, solution of (9) is written as

$$
y(t)=\int_{0}^{\omega} G_{1}(t, s) h(s) d s,
$$

where

$$
G_{1}(t, s)= \begin{cases}\frac{e^{-\rho(t-s)}}{1-e^{-\omega \rho}}, & 0 \leq s \leq t \leq \omega, \\ \frac{e^{-\rho(\omega+t-s)}}{1-e^{-\omega \rho}}, & 0 \leq t<s \leq \omega .\end{cases}
$$

Solution of (10) is written as

$$
x(t)=\int_{0}^{\omega} G_{2}(t, s) y(s) d s .
$$
[20].

Next, we will consider $G_{2}(t, s)$, which can be found in Suppose that

$$
\frac{1}{Q \omega}\left[\exp \left(\int_{0}^{\omega} p(u) d u\right)-1\right] \geq 1
$$

where

$$
\begin{gathered}
R=\max _{t \in[0, \omega]}\left|\int_{0}^{\omega} \frac{\exp \left(\int_{t}^{s} p(u) d u\right)}{\exp \left(\int_{0}^{\omega} p(u) d u\right)-1} q(s) d s\right|, \\
Q=\left[1+\exp \left(\int_{0}^{\omega} p(u) d u\right)\right]^{2} R .
\end{gathered}
$$


Then there are continuous $\omega$-periodic functions $\alpha$ and $\beta$ such that $\beta(t)>0, \int_{0}^{\omega} \alpha(u) d u>0$ and

$$
\alpha(t)+\beta(t)=p(t), \quad \beta^{\prime}(t)+\alpha(t) \beta(t)=q(t), \quad \text { for } t \in \mathbb{R} .
$$

Suppose (14) holds and $y \in X$; then the equation

$$
x^{\prime \prime}+p(t) x^{\prime}+q(t) x=y(t)
$$

has an $\omega$-periodic solution. Moreover, the periodic solution can be expressed by

$$
x(t)=\int_{0}^{\omega} G_{2}(t, s) y(s) d s,
$$

where

$$
\begin{aligned}
G_{2}(t, s)= & \left(\int_{t}^{s} \exp \left[\int_{t}^{u} \beta(v) d v+\int_{u}^{s} \alpha(v) d v\right] d u\right. \\
& \left.+\int_{s}^{t+\omega} \exp \left[\int_{t}^{u} \beta(v) d v+\int_{u}^{s+\omega} \alpha(v) d v\right] d u\right) \\
& \times\left(\left[\exp \left(\int_{0}^{\omega} \alpha(u) d u\right)-1\right]\right. \\
& \left.\times\left[\exp \left(\int_{0}^{\omega} \beta(u) d u\right)-1\right]\right)^{-1},
\end{aligned}
$$

with

$$
\begin{gathered}
G_{2}(t, t+\omega)=G_{2}(t, t), \quad G_{2}(t+\omega, s+\omega)=G_{2}(t, s), \\
\frac{\partial}{\partial s} G_{2}(t, s)=\alpha(s) G_{2}(t, s)-\frac{\exp \left(\int_{t}^{s} \beta(v) d v\right)}{\exp \left(\int_{0}^{\omega} \beta(v) d v\right)-1}, \\
\frac{\partial}{\partial t} G_{2}(t, s)=-\beta(t) G_{2}(t, s)+\frac{\exp \left(\int_{t}^{s} \alpha(v) d v\right)}{\exp \left(\int_{0}^{\omega} \alpha(v) d v\right)-1} .
\end{gathered}
$$

If

Let $A=\int_{0}^{\omega} p(u) d u$ and $B=\omega^{2} \exp \left((1 / \omega) \int_{0}^{\omega} \ln q(u) d u\right)$.

$$
A^{2} \geq 4 B
$$

then we have

$$
\begin{aligned}
& \min \left\{\int_{0}^{\omega} \alpha(u) d u, \int_{0}^{\omega} \beta(u) d u\right\} \geq \frac{1}{2}\left(A-\sqrt{A^{2}-4 B}\right):=l, \\
& \max \left\{\int_{0}^{\omega} \alpha(u) d u, \int_{0}^{\omega} \beta(u) d u\right\} \leq \frac{1}{2}\left(A+\sqrt{A^{2}-4 B}\right):=m .
\end{aligned}
$$

Moreover,

$$
0<C:=\frac{\omega}{\left(e^{m}-1\right)^{2}} \leq G_{2}(t, s) \leq \frac{\omega \exp \left(\int_{0}^{\omega} p(u) d u\right)}{\left(e^{l}-1\right)^{2}}:=D .
$$

Therefore, we know that the solution of (8) is written as

$$
\begin{aligned}
x(t) & =\int_{0}^{\omega} G_{2}(t, \tau) \int_{0}^{\omega} G_{1}(\tau, s) h(s) d s d \tau \\
& =\int_{0}^{\omega} \int_{0}^{\omega} G_{2}(t, \tau) G_{1}(\tau, s) h(s) d s d \tau \\
& =\int_{0}^{\omega}\left[\int_{0}^{\omega} G_{2}(t, s) G_{1}(s, \tau) d s\right] h(\tau) d \tau \\
& =\int_{0}^{\omega}\left[\int_{0}^{\omega} G_{2}(t, \tau) G_{1}(\tau, s) d \tau\right] h(s) d s .
\end{aligned}
$$

Therefore, by writing

$$
G^{1}(t, s)=\int_{0}^{\omega} G_{2}(t, \tau) G_{1}(\tau, s) d \tau
$$

we can get

$$
x(t)=\int_{0}^{\omega} G^{1}(t, s) h(s) d s .
$$

Lemma 1. Assume that (14) and (21) hold. Then $G^{1}(t, s)>0$ for all $(t, s) \in[0, \omega] \times[0, \omega]$.

Proof. From (23), we know $G_{2}(t, s)>0$. Since $G_{1}(t, s)>0$, from $(25)$ we see that $G^{1}(t, s)>0$ for all $(t, s) \in[0, \omega] \times[0, \omega]$.

Case 2. There exist an $\omega$-periodic differentiable function $m$ and a positive real constant $\rho$ such that $a(t)=\rho, b(t)=m(t)$, and $c(t)=\rho m(t)+m^{\prime}(t)$. Then, (8) is transformed into

$$
\begin{gathered}
y^{\prime}(t)+\rho y(t)=h(t), \\
y(0)=y(\omega), \\
x^{\prime \prime}(t)+m(t) x(t)=y(t), \\
x(0)=x(\omega), \quad x^{\prime}(0)=x^{\prime}(\omega) .
\end{gathered}
$$

Then, solution of (27) is written as

$$
y(t)=\int_{0}^{\omega} G_{1}(t, s) h(s) d s .
$$

Solution of (28) is written as

$$
x(t)=\int_{0}^{\omega} G_{3}(t, s) y(s) d s .
$$

By the following lemma, which can be found in [21], we will consider the sign of $G_{3}(t, s)$.

Lemma 2 (see [21]). Let us define

$$
K(q)= \begin{cases}\frac{2 \pi}{q \omega^{1+2 / q}}\left(\frac{2}{2+q}\right)^{1-2 / q}, & \text { if } 1 \leq q<\infty, \\ \times\left(\frac{\Gamma(1 / q)}{\Gamma((1 / 2)+(1 / q))}\right)^{2} & \\ \frac{4}{\omega}, & \text { if } q=\infty,\end{cases}
$$


where $\Gamma$ is the Gamma function. Assume that $m(t)>0$ and $m \in L^{p}(0, T)$ for some $1 \leq p \leq \infty$. If

$$
\|m\|_{p} \leq K\left(2 p^{*}\right),
$$

where $p^{*}=p /(p-1)$ if $1 \leq q<\infty$ and $p^{*}=1$ if $p=+\infty$, then $G_{3}(t, s) \geq 0$ for all $(t, s) \in[0, \omega] \times[0, \omega]$.

Similarly to (26), we know that the solution of (8) can be written as

$$
x(t)=\int_{0}^{\omega} G^{2}(t, s) h(s) d s .
$$

Here, $G^{2}(t, s)=\int_{0}^{\omega} G_{3}(t, \tau) G_{1}(\tau, s) d \tau$. And we get the following Lemma.

Lemma 3. Assume $m \in L^{p}(0, T)$ for some $1 \leq p \leq \infty$. $m(t)>$ 0 and (32) hold. Then, $G^{2}(t, s) \geq 0$ for all $(t, s) \in[0, \omega] \times[0, \omega]$.

Proof. From Lemma 10, we know $G_{2}(t, s)>0$. Since $G_{1}(t, s)>$ 0 , from (25) we see that $G^{2}(t, s)>0$ for all $(t, s) \in[0, \omega] \times$ $[0, \omega]$.

\section{Preliminary Lemmas}

Firstly, we establish the existence of positive periodic solutions for third-order differential equation (6) by using fixed point theorem, which can be found in [22].

Lemma 4 (see [22]). Let $X$ be a Banach space and $K$ a cone in $X$. Assume that $\Omega_{1}, \Omega_{2}$ are bounded open subsets of $X$ with $0 \in \Omega_{1}, \bar{\Omega}_{1} \subset \Omega_{2}$, and let

$$
T: K \cap\left(\bar{\Omega}_{2} \backslash \Omega_{1}\right) \longrightarrow K
$$

be a completely continuous operator such that either

(i) $\|T u\| \geq\|u\|, u \in K \cap \partial \Omega_{1}$ and $\|T u\| \leq\|u\|, u \in K \cap$ $\partial \Omega_{2}$; or

(ii) $\|T u\| \leq\|u\|, u \in K \cap \partial \Omega_{1}$ and $\|T u\| \geq\|u\|, u \in K \cap$ $\partial \Omega_{2}$.

Then $T$ has a fixed point in $K \cap\left(\bar{\Omega}_{2} \backslash \Omega_{1}\right)$.

For the sake of convenience, we list the following assumptions which will be used repeatedly in the sequel:

$\left(H_{1}\right) f(x)$ is a scalar continuous function defined for $x>0$, and $f(x)>0$ for $x>0$.

$$
\begin{aligned}
& \left(H_{2}\right) g(t) \geq 0, t \in[0, \omega], \int_{0}^{\omega} g(t) d t>0 . \\
& \left(H_{3}\right) g(t)>0 \text { for } t \in[0, \omega] .
\end{aligned}
$$

Under Lemmas 1 and 3, we always denote

$$
\begin{aligned}
& m_{i}=\min _{0 \leq s, t \leq \omega} G^{i}(t, s), \quad M_{i}=\max _{0 \leq s, t \leq \omega} G^{i}(t, s), \quad \sigma_{i}=\frac{m_{i}}{M_{i}}, \\
& i=1,2 \text {. }
\end{aligned}
$$

Obviously, $M_{i}>m_{i}>0$ and $0<\sigma_{i}<1$.
Case 1. Let $a(t)=\rho+p(t), b(t)=\rho p(t)+q(t)+p^{\prime}(t), c(t)=$ $\rho q(t)+q^{\prime}(t)$. The following are the main existence results in this section.

Define the cone $K$ in $X$ by

$$
\begin{gathered}
K=\{x \in X: x(t) \geq 0 \forall t \in[0, \omega], \\
\left.\min _{t \in R} x(t) \geq \sigma_{1}\|x\|\right\} .
\end{gathered}
$$

We take $X=C_{\omega}$ with $\|x\|=\max _{t}|x(t)|$. Also, for $r>0$, let

$$
\Omega_{r}=\{x \in K:\|x\|<r\} .
$$

Define the operator $T: K \backslash\{0\} \rightarrow X$ as follows:

$$
\left(T_{\mu} x\right)(t)=\mu \int_{0}^{\omega} G^{1}(t, s)(g(s) f(x(s))+e(s)) d s,
$$

where $e$ is nonnegative and $g(s) f(x(s))+e(s)$ is nonnegative. If $e$ takes negative values, we will choose $x(s)$ so that $g(s) f(x(s))+e(s)$ is nonnegative. This is possible because $\lim _{x \rightarrow 0} f(x)=\infty$ or $\lim _{|x| \rightarrow \infty} f(x)=\infty$.

Now, if $x$ is a fixed point of $T_{\mu}$ in $K \backslash\{0\}$, then $x$ is a positive solution of (6). Also note that each component $x(t)$ of any nonnegative periodic solution $x$ is strictly positive for all $t$ because of the positiveness of the Green functions and assumptions $\left(H_{1}\right)$ and $\left(H_{2}\right)$. We now look at several properties of the operator.

Lemma 5. Assume that (14), (21), $\left(H_{1}\right)$, and $\left(H_{2}\right)$ hold and $e(t) \geq 0, t \in[0, \omega]$. Then, $T_{\mu}(K \backslash\{0\}) \subset K$ and $T_{\mu}: K \backslash\{0\} \rightarrow$ $K$ is completely continuous.

Proof. If $x \in K \backslash\{0\}$, then $\min _{t \in[0, \omega]} x(t) \geq \sigma_{1}\|x\|>0$, and then $T_{\mu}$ is defined. Now we have that

$$
\begin{aligned}
\min _{t \in[0, \omega]} T_{\mu} x(t) & \geq m_{1} \mu \int_{0}^{\omega}(g(s) f(x(s))+e(s)) d s \\
& =\mu \sigma_{1} M_{1} \int_{0}^{\omega}(g(s) f(x(s))+e(s)) d s \\
& \geq \sigma_{1} \sup _{t \in[0, \omega]} T_{\mu} x(t)=\sigma_{1}\left\|T_{\mu} x\right\| .
\end{aligned}
$$

Thus, $T_{\mu}(K \backslash\{0\}) \subset K$. It is easy to verify that $T_{\mu}$ is completely continuous.

If $e(t)$ takes negative values, we need to choose appropriate domains so that $g(s) f(x(s))+e(s)$ become nonnegative. The proof of $T_{\mu}(K \backslash\{0\}) \subset K$ and $T_{\mu}\left(K \backslash \Omega_{R}\right) \subset K$ in Lemma 6 is the same as in Lemma 5.

Lemma 6. Assume that (14), (21), $\left(H_{1}\right)$, and $\left(H_{3}\right)$ hold.

(a) If $\lim _{x \rightarrow 0} f(x)=\infty$, there is a $\delta>0$ such that if $0<$ $r<\delta$, then $T_{\mu}$ is defined on $\bar{\Omega}_{r} \backslash\{0\}, T_{\mu}\left(\bar{\Omega}_{r} \backslash\{0\}\right) \subset K$, and $T_{\mu}: \bar{\Omega}_{r} \backslash\{0\} \rightarrow K$ is completely continuous.

(b) If $\lim _{x \rightarrow \infty} f(x)=\infty$, there is a $\Delta>0$ such that if $R>\Delta$, then $T_{\mu}$ is defined on $K \backslash \Omega_{R}, T_{\mu}\left(K \backslash \Omega_{R}\right) \subset K$, and $T_{\mu}: K \backslash \Omega_{R} \rightarrow K$ is completely continuous. 
Proof. We split $g(t) f(x(t))+e(t)$ into the two terms $(1 / 2) g(t) f(x(t)$ and $(1 / 2) g(t) f(x(t))+e(t)$. The first term is always nonnegative and used to carry out the estimates of the operator in the lemma and corollaries in this section. We will make the second term $(1 / 2) g(t) f(x(t))+e(t)$ nonnegative by choosing appropriate domains of $f$. The choice of the even split of $g(t) f(x(t))$ here is not necessarily optimal in terms of obtaining maximal $\mu$-intervals for the existence of periodic solutions of the equation.

Noting that $g(t)$ is positive on $[0, \omega], \lim _{x \rightarrow 0} f(x)=\infty$, implies that there exists a constant $\delta>0$ such that

$$
f(x) \geq 2 \frac{\max _{t \in[0, \omega]}\{e(t)+1\}}{\min _{t \in[0, \omega]} g(t)},
$$

for $0<x<\delta$. Now for $x \in \bar{\Omega}_{r} \backslash\{0\}$ and $0<r<\delta$, note that

$$
\delta>r \geq x(t) \geq \min _{t \in[0, \omega]} x(t) \geq \sigma_{1}\|x\|>0, \quad t \in[0, \omega],
$$

and, therefore, we have, for $t \in[0, \omega]$,

$$
\begin{aligned}
g(t) f(x(t))+e(t) & \geq \frac{1}{2} g(t) f(x(t))+e(t) \\
& \geq g(t) \frac{\max _{t \in[0, \omega]}\{e(t)+1\}}{\min _{t \in[0, \omega]} g(t)}+e(t)>0 .
\end{aligned}
$$

Thus, it is clear that $T_{\mu} x(t)$ in (38) is well defined and positive, and now it is easy to see that $T_{\mu}\left(\bar{\Omega}_{r} \backslash\{0\}\right) \subset K$ and $T_{\mu}: \bar{\Omega}_{r} \backslash\{0\} \rightarrow K$ is completely continuous.

On the other hand, if $\lim _{x \rightarrow \infty} f(x)=\infty$, there is an $R^{\prime \prime}>$ 0 such that

$$
f(x) \geq 2 \frac{\max _{t \in[0, \omega]}\{e(t)+1\}}{\min _{t \in[0, \omega]} g(t)},
$$

for $|x| \geq R^{\prime \prime}$. Now let $\Delta=R^{\prime \prime} / \sigma_{1}$. Then for $x \in K \backslash \Omega_{R}, R>\Delta$, we have that $\min _{t \in[0, \omega]} x(t) \geq \sigma_{1}\|x\|>R^{\prime \prime}$, and, therefore,

$$
\begin{array}{r}
g(t) f(x(t))+e(t) \geq \frac{1}{2} g(t) f(x(t))+e(t)>0, \\
t \in[0, \omega] .
\end{array}
$$

Now, $T_{\mu} x(t)$ in (38) is well defined and positive. It is clear that $T_{\mu}\left(K \backslash \Omega_{R}\right) \subset K$ and $T_{\mu}: K \backslash \Omega_{R} \rightarrow K$ is completely continuous.

Now let

$$
\Gamma=\frac{1}{2} m_{1} \sigma_{1} \int_{0}^{\omega} g(s) d s .
$$

Lemma 7. Assume that (14), (21), $\left(H_{1}\right)$, and $\left(H_{2}\right)$ hold and $e(t) \geq 0, t \in[0, \omega]$. Let $r>0$ and if there exist $\eta>0$ such that

$$
f(x(t)) \geq \eta x(t) \quad \text { for } t \in[0, \omega],
$$

for $x(t) \in \partial \Omega_{r}$, then the following inequality holds:

$$
\left\|T_{\mu} x\right\| \geq \mu \Gamma \eta\|x\| \text {. }
$$

Proof. From the definition of $T_{\mu} x$, it follows that

$$
\begin{aligned}
\left\|T_{\mu} x\right\| & \geq \max _{t \in[0, \omega]} T_{\mu} x(t) \\
& \geq \frac{1}{2} \mu m_{1} \int_{0}^{\omega} g(s) f(x(s)) d s \\
& \geq \frac{1}{2} \mu m_{1} \int_{0}^{\omega} g(s) \eta x(s) d s \\
& \geq \frac{1}{2} \mu m_{1} \sigma_{1} \int_{0}^{\omega} g(s) d s \eta\|x\|=\mu \Gamma \eta\|x\| .
\end{aligned}
$$

If $e(t)$ takes negative values, we need to adjust $\delta$ and $\Delta$ in Lemma 6 to guarantee that $g(t) f(x(t))+e(t)$ is nonnegative.

Corollary 8. Assume that (14), (21), $\left(H_{1}\right)$, and $\left(H_{3}\right)$ hold.

(a) If $\lim _{x \rightarrow 0} f(x)=\infty$, then Lemma 7 holds assuming that $0<r<\delta$, where $\delta$ is defined by Lemma 6 .

(b) If $\lim _{x \rightarrow \infty} f(x)=\infty$, then Lemma 7 holds assuming that $\Delta>0$, where $\Delta$ is defined by Lemma 6 .

Proof. We split $g(t) f(x(t))+e(t)$ into the two terms $(1 / 2) g(t) f(x(t))$ and $(1 / 2) g(t) f(x(t))+e(t)$. By choosing $\delta$ and $\Delta$ in Lemma $6, g(t) f(x(t))+e(t)$ become nonnegative. The estimate in Corollary 8 can be carried out by the first terms as in Lemma 7.

Let $\widehat{f}(\theta):[1, \infty) \rightarrow \mathbb{R}_{+}$be the function given by

$$
\widehat{f}(\theta)=\max \left\{f(u): u \in \mathbb{R}_{+}, 1 \leq u \leq \theta\right\} .
$$

It is easy to see that $\widehat{f}(\theta)$ is a nondecreasing function on $[1, \infty)$. The following lemma is essentially the same as Lemma 2.8 in [23].

Lemma 9 (see [23]). Assume $\left(H_{1}\right)$ holds. If $\lim _{|x| \rightarrow \infty}(f(x) /|x|)$ exists (which can be infinity), then $\lim _{\theta \rightarrow \infty}(\widehat{f}(\theta) / \theta)$ exists and $\lim _{\theta \rightarrow \infty}(\widehat{f}(\theta) / \theta)=$ $\lim _{|x| \rightarrow \infty}(f(x) /|x|)$.

Lemma 10. Assume that (14), (21), $\left(H_{1}\right)$, and $\left(H_{2}\right)$ hold and $e(t) \geq 0, t \in[0, \omega]$. Let $r>\max \left\{1 / \sigma_{1}, 2 \mu M_{1} \int_{0}^{\omega}|e(s)| d s\right\}$ and if there exists an $\varepsilon>0$ such that

$$
\widehat{f}(r) \leq \varepsilon r
$$

then

$$
\left\|T_{\mu} x\right\| \leq \mu \widehat{C} \varepsilon\|x\|+\frac{1}{2}\|x\| \quad \text { for } x \in \partial \Omega_{r}
$$

where the constant $\widehat{C}=M_{1} \int_{0}^{\omega} g(s) d s$. 
Proof. From the definition of $T_{\mu}$, we have, for $x \in \partial \Omega_{r}$,

$$
\begin{aligned}
\left\|T_{\mu} x\right\| & =\max _{t \in[0, \omega]} T_{\mu} x(t) \\
& \leq \mu M_{1} \int_{0}^{\omega} g(s) f(x(s)) d s+\mu M_{1} \int_{0}^{\omega}|e(s)| d s \\
& \leq \mu M_{1} \int_{0}^{\omega} g(s) \hat{f}(r) d s+\frac{r}{2} \\
& \leq \mu M_{1} \int_{0}^{\omega} g(s) d s r \varepsilon+\frac{r}{2} \\
& =\mu \widehat{C} \varepsilon\|x\|+\frac{1}{2}\|x\| .
\end{aligned}
$$

If $e(t)$ takes negative values, we need to restrict the domain of $T_{\mu}$ to guarantee that $g(t) f(x(t))+e(t)$ is nonnegative.

Corollary 11. Assume that (14), (21), $\left(H_{1}\right)$, and $\left(H_{3}\right)$ hold. If $\lim _{x \rightarrow \infty} f(x)=\infty$, Lemma 10 holds assuming that $r>\Delta$, where $\Delta$ is defined by Lemma 6.

Proof. If we choose $\Delta$ defined in Lemma 6, then $T_{\mu}$ is well defined and $g(t) f(x(t))+e(t)$ is nonnegative, and Corollary 11 can be shown in the same way as Lemma 10.

The conclusions of Lemmas 7 and 10 are based on the inequality assumptions between $f(x)$ and $x$. If these assumptions are not necessarily true, we will have the following results.

Lemma 12. Assume that (14), (21), $\left(H_{1}\right)$, and $\left(H_{2}\right)$ hold and $e(t) \geq 0, t \in[0, \omega]$. Let $r>0$. Then

$$
\left\|T_{\mu} x\right\| \geq \mu \frac{m_{1} \widehat{m}_{r 1}}{2} \int_{0}^{\omega} g(s) d s,
$$

for all $x \in \partial \Omega_{r}$, where $\widehat{m}_{r 1}=\min \left\{f(x): x \in \mathbb{R}_{+}, \sigma_{1} r \leq x \leq\right.$ $r\}>0$.

Proof. If $x(t) \in \partial \Omega_{r}$, then $\sigma_{1} r \leq x(t) \leq r$, for $t \in[0, \omega]$. Therefore $f(x(t)) \geq \widehat{m}_{r 1}$ for $t \in[0, \omega]$. By the definition of $T_{\mu}$, we have

$$
\begin{aligned}
\left\|T_{\mu} x\right\| & =\max _{t \in[0, \omega]} T_{\mu} x(t) \\
& \geq \frac{1}{2} \mu m_{1} \int_{0}^{\omega} g(s) f(x(s)) d s \\
& \geq \mu \frac{m_{1} \widehat{m}_{r 1}}{2} \int_{0}^{\omega} g(s) d s .
\end{aligned}
$$

Now we consider the cases that $e(t)$ may take negative values. We need to restrict the domain of $T_{\mu}$ to guarantee that $g(t) f(x(t))+e(t)$ is nonnegative. $(1 / 2) g(t) f(x(t))$ is used to carry out the estimates as Lemma 12.
Corollary 13. Assume that (14), (21), $\left(H_{1}\right)$, and $\left(H_{3}\right)$ hold.

(a) If $\lim _{x \rightarrow 0} f(x)=\infty$, then Lemma 12 holds assuming that $0<r<\delta$, where $\delta>0$ is defined by Lemma 6 .

(b) If $\lim _{x \rightarrow \infty} f(x)=\infty$, then Lemma 12 holds assuming that $r>\Delta$, where $\Delta$ is defined by Lemma 6 .

Proof. By selecting $\delta$ and $\Delta$ defined in Lemma $6, T_{\mu}$ is well defined and $g(t) f(x(t))+e(t)$ is nonnegative, and then Corollary 13 can be shown as Lemma 12.

Lemma 14. Assume that (14), (21), $\left(H_{1}\right)$, and $\left(H_{2}\right)$ hold and $e(t) \geq 0, t \in[0, \omega] ;$ let $r>0$. Then

$$
\left\|T_{\mu} x\right\| \leq \mu\left(M_{1} \int_{0}^{\omega} g(s) \widehat{M}_{r 1} d s+M_{1} \int_{0}^{\omega}|e(s)| d s\right),
$$

for all $x \in \partial \Omega_{r}$, where $\widehat{M}_{r 1}=\max \left\{f(x): x \in \mathbb{R}_{+}, \sigma_{1} r \leq x \leq\right.$ $r\}>0$.

Proof. If $x \in \partial \Omega_{r}$, then $\sigma_{1} r \leq x(t) \leq r, t \in[0, \omega]$. Therefore, $f(x(t)) \leq \widehat{M}_{r 1}$ for $t \in[0, \omega]$. Thus we have that

$$
\begin{aligned}
\left\|T_{\mu} x\right\| & =\max _{t \in[0, \omega]} T_{\mu} x(t) \\
& \leq \mu M_{1} \int_{0}^{\omega} g(s) f(x(s)) d s+\mu M_{1} \int_{0}^{\omega} e(s) d s \\
& \leq \mu M_{1} \int_{0}^{\omega} g(s) f(x(s)) d s+\mu M_{1} \int_{0}^{\omega}|e(s)| d s \\
& \leq \mu M_{1} \int_{0}^{\omega} g(s) \widehat{M}_{r 1} d s+\mu M_{1} \int_{0}^{\omega}|e(s)| d s \\
& =\mu\left(M_{1} \int_{0}^{\omega} g(s) \widehat{M}_{r 1} d s+M_{1} \int_{0}^{\omega}|e(s)| d s\right) .
\end{aligned}
$$

Again, if $e(t)$ takes negative values, we need to restrict $r$ and $R$ to guarantee $g(t) f(x(t))+e(t)$ is nonnegative.

Corollary 15. Assume that (14), (21), $\left(H_{1}\right)$, and $\left(H_{3}\right)$ hold.

(a) If $\lim _{x \rightarrow 0} f(x)=\infty$, then Lemma 14 holds assuming that $0<r<\delta$, where $\delta>0$ is defined by Lemma 6 .

(b) If $\lim _{x \rightarrow \infty} f(x)=\infty$, then Lemma 14 holds assuming that $r>\Delta$, where $\Delta$ is defined by Lemma 6 .

Proof. By selecting $\delta$ and $\Delta$ defined in Lemma $6, T_{\mu}$ is well defined and $g(t) f(x(t))+e(t)$ is nonnegative, and then Corollary 15 can be shown as Lemma 14 .

\section{Main Results}

In this section, we present out main results for the existence and multiplicity of positive periodic solutions of singular third-order equation of repulsive type (6). We state our theorems as follows.

Theorem 16. Let (14), (21), $\left(H_{1}\right)$, and $\left(H_{2}\right)$ hold and $e(t) \geq$ $0, t \in[0, \omega]$. Assume that $\lim _{x \rightarrow 0} f(x)=\infty$. 
(a) If $\lim _{x \rightarrow \infty}(f(x) / x)=0$, then, for all $\mu>0$, (6) has a positive periodic solution.

(b) If $\lim _{x \rightarrow \infty}(f(x) / x)=\infty$, then, for all sufficiently small $\mu>0$, (6) has two positive periodic solutions.

(c) There exists a $\mu_{1}$ such that (6) has a positive periodic solution for $0<\mu<\mu_{1}$.

Proof. (a) Since $e(t) \geq 0, T_{\mu}$ is defined on $K \backslash\{0\}$ and $g(t) f(x(t))+e(t)$ is nonnegative. Noting $\lim _{x \rightarrow \infty}(f(x) / x)=$ 0 , it follows from Lemma 9 that $\lim _{\theta \rightarrow \infty}(\widehat{f}(\theta) / \theta)=0$. Therefore, we can choose $r_{1}>\max \left\{1 / \sigma_{1}, 2 \mu M_{1} \int_{0}^{\omega}|e(s)| d s\right\}$ so that $\widehat{f}\left(r_{1}\right) \leq \varepsilon r_{1}$, where the constant $\varepsilon>0$ satisfies

$$
\mu \widehat{C} \varepsilon<\frac{1}{2}
$$

and $\widehat{C}$ is the positive constant defined in Lemma 10. We have by Lemma 10 that

$$
\left\|T_{\mu} x\right\| \leq\left(\mu \widehat{C} \varepsilon+\frac{1}{2}\right)\|x\|<\|x\| \quad \text { for } x \in \partial \Omega_{r_{1}} .
$$

On the other hand, by the condition $\lim _{x \rightarrow 0} f(x)=\infty$, there is a positive number $r_{2}<r_{1}$ such that

$$
f(x) \geq \eta x,
$$

for $x \in \mathbb{R}_{+} \backslash\{0\}$ and $x \leq r_{2}$, where $\eta>0$ is chosen so that

$$
\mu \Gamma \eta>1 \text {. }
$$

It is easy to see that, for $x \in \partial \Omega_{r_{2}}, t \in[0, \omega]$,

$$
f(x) \geq \eta x(t) .
$$

Lemma 7 implies that

$$
\left\|T_{\mu} x\right\| \geq \mu \Gamma \eta\|x\|>\|x\| \quad \text { for } x \in \partial \Omega_{r_{2}} .
$$

By Lemma $4, T_{\mu}$ has a fixed point $x \in \bar{\Omega}_{r_{1}} \backslash \Omega_{r_{2}}$. The fixed point $x \in \bar{\Omega}_{r_{1}} \backslash \Omega_{r_{2}}$ is the desired positive periodic solution of (6).

(b) Again, since $e(t) \geq 0, T_{\mu}$ is defined on $K \backslash\{0\}$ and $g(t) f(x(t))+e(t)$ is nonnegative. Fix two numbers $0<r_{3}<r_{4}$; there exists a $\mu_{0}>0$ such that

$$
\begin{aligned}
& \mu_{0}<\frac{r_{3}}{M_{1} \int_{0}^{\omega} g(s) \widehat{M}_{r_{31}} d s+M_{1} \int_{0}^{\omega}|e(s)| d s}, \\
& \mu_{0}<\frac{r_{4}}{M_{1} \int_{0}^{\omega} g(s) \widehat{M}_{r_{41}} d s+M_{1} \int_{0}^{\omega}|e(s)| d s},
\end{aligned}
$$

where $\widehat{M}_{r_{31}}$ and $\widehat{M}_{r_{41}}$ are defined in Lemma 14, which implies that, for $0<\mu<\mu_{0}$,

$$
\left\|T_{\mu} x\right\|<\|x\| \quad \text { for } x \in \partial \Omega_{r_{j}}(j=3,4) .
$$

On the other hand, in view of the assumptions $\lim _{x \rightarrow \infty}(f(x) / x)=\infty$ and $\lim _{x \rightarrow 0} f(x)=\infty$, there are positive numbers $0<r_{2}<r_{3}<r_{4}<r_{1}^{\prime}$ such that

$$
f(x) \geq \eta x
$$

for $x \in \mathbb{R}_{+}$and $0<x \leq r_{2}$ or $x>r_{1}^{\prime}$ where $\eta>0$ is chosen so that

$$
\mu \Gamma \eta>1
$$

Thus if $x \in \partial \Omega_{r_{2}}$, then

$$
f(x(t)) \geq \eta x(t), \quad t \in[0, \omega] .
$$

Let $r_{1}=r_{1}^{\prime} / \sigma_{1}$. If $x \in \partial \Omega_{r_{1}}$, then

$$
\min _{t \in[0, \omega]} x(t) \geq \sigma_{1}\|x\|=\sigma_{1} r_{1} \geq r_{1}^{\prime}
$$

which implies that

$$
f(x(t)) \geq \eta x(t) \quad \text { for } t \in[0, \omega] .
$$

Thus, Lemma 7 implies that

$$
\begin{array}{ll}
\left\|T_{\mu} x\right\| \geq \mu \Gamma \eta\|x\|>\|x\| & \text { for } x \in \partial \Omega_{r_{1}}, \\
\left\|T_{\mu} x\right\| \geq \mu \Gamma \eta\|x\|>\|x\| & \text { for } x \in \partial \Omega_{r_{2}} .
\end{array}
$$

It follows from Lemma 4 that $T_{\mu}$ has two fixed points $x_{1}(t)$ and $x_{2}(t)$ such that $x_{1}(t) \in \bar{\Omega}_{r_{3}} \backslash \Omega_{r_{2}}$ and $x_{2}(t) \in \bar{\Omega}_{r_{1}} \backslash \Omega_{r_{4}}$, which are the desired distinct positive periodic solutions of (6) for $\mu<\mu_{0}$ satisfying

$$
r_{2}<\left\|x_{1}\right\|<r_{3}<r_{4}<\left\|x_{2}\right\|<r_{1} .
$$

(c) First we note that $T_{\mu}$ is defined on $K \backslash\{0\}$ and $g(t) f(x(t))+e(t)$ is nonnegative since $e(t) \geq 0$. Fix a number $r_{3}>0$. Lemma 14 implies that there exists a $\mu_{1}>0$ such that we have, for $0<\mu<\mu_{1}$,

$$
\left\|T_{\mu} x\right\|<\|x\| \quad \text { for } x \in \partial \Omega_{r_{3}} .
$$

On the other hand, in view of the assumption $\lim _{x \rightarrow 0} f(x)=\infty$, there is a positive number $0<r_{2}<r_{3}$ such that

$$
f(x) \geq \eta x
$$

for $x \in \mathbb{R}_{+}$and $0<x \leq r_{2}$, where $\eta>0$ is chosen so that

$$
\mu \Gamma \eta>1 \text {. }
$$

Thus, if $x \in \partial \Omega_{r_{2}}$, then

$$
f(x(t)) \geq \eta x(t), \quad t \in[0, \omega] .
$$

Thus, Lemma 7 implies that

$$
\left\|T_{\mu} x\right\| \geq \mu \Gamma \eta\|x\|>\|x\| \quad \text { for } x \in \partial \Omega_{r_{2}} .
$$

Lemma 4 implies that $T_{\mu}$ has a fixed point $x \in \bar{\Omega}_{r_{3}} \backslash \Omega_{r_{2}}$. The fixed point $x \in \bar{\Omega}_{r_{3}} \backslash \Omega_{r_{2}}$ is the desired positive periodic solution of (6).

When $e(t)$ takes negative values, we give the following theorem. 
Theorem 17. Let (14), (21), $\left(H_{1}\right)$, and $\left(H_{3}\right)$ hold. Assume that $\lim _{x \rightarrow 0} f(x)=\infty$.

(a) If $\lim _{x \rightarrow \infty} f(x)=\infty$ and $\lim _{x \rightarrow \infty}(f(x) / x)=0$, then there exists $\mu_{0}>0$ such that (6) has a positive periodic solution for $\mu>\mu_{0}$.

(b) If $\lim _{x \rightarrow \infty}(f(x) / x)=\infty$, then, for all sufficiently small $\mu>0$, (6) has two positive periodic solutions.

(c) There exists a $\mu_{1}>0$ such that (6) has a positive periodic solution for $0<\mu<\mu_{1}$.

Proof. (a) Since $\lim _{|x| \rightarrow \infty} f(x)=\infty$, by Lemma 6 , there is a $\Delta>0$ such that if $R>\Delta$; then $g(t) f(x(t))+e(t)$ is nonnegative and $T_{\mu}: K \backslash \Omega_{R} \rightarrow K$ is defined. Now, for a fixed number $r_{1}>\Delta$, Corollary 13 implies that there exists a $\mu_{0}>0$ such that, for $\mu>\mu_{0}$,

$$
\left\|T_{\mu} x\right\|>\|x\| \quad \text { for } x \in \partial \Omega_{r_{1}} .
$$

On the other hand, since $\lim _{|x| \rightarrow \infty}(f(x) / x)=0$, it follows from Lemma 2 that $\lim _{\theta \rightarrow \infty}(\widehat{f}(\theta) / \theta)=0$. Therefore, we can choose

$$
r_{2}>\max \left\{2 r_{1}, \frac{1}{\sigma_{1}}, 2 \mu M_{1} \int_{0}^{\omega}|e(s)| d s\right\}>\Delta,
$$

so that $\widehat{f}\left(r_{2}\right) \leq \varepsilon r_{2}$, where the constant $\varepsilon>0$ satisfies

$$
\mu \widehat{C} \varepsilon<\frac{1}{2}
$$

We have, by Corollary 11, that

$$
\left\|T_{\mu} x\right\| \leq\left(\mu \widehat{C} \varepsilon+\frac{1}{2}\right)\|x\|<\|x\| \quad \text { for } x \in \partial \Omega_{r_{2}} .
$$

By Lemma $4, T_{\mu}$ has a fixed point $x \in \bar{\Omega}_{r_{2}} \backslash \Omega_{r_{1}}$. The fixed point $x \in \bar{\Omega}_{r_{2}} \backslash \Omega_{r_{1}}$ is the desired positive periodic solution of (6).

(b) First, since $\lim _{x \rightarrow 0} f(x)=\infty$, by Lemma 6, there is $\delta>0$ such that if $0<r<\delta, T_{\mu}$ is defined on $\widehat{\Omega} \backslash\{0\}$ and $g(t) f(x(t))+e(t)$ is nonnegative. Furthermore, $T_{\mu}\left(\widehat{\Omega}_{r} \backslash\{0\}\right) \subset$ $K$. Now for a fixed number $r_{1}<\delta$, Corollary 15 implies that there exists a $\mu_{1}>0$ such that we have, for $\mu<\mu_{1}$,

$$
\left\|T_{\mu} x\right\|<\|x\| \quad \text { for } x \in \partial \Omega_{r_{1}} .
$$

In view of the assumption $\lim _{x \rightarrow 0} f(x)=\infty$, there is a positive number $0<r_{3}<r_{1}$ such that

$$
f(x) \geq \eta x
$$

for $x \in \mathbb{R}_{+}$and $0<x \leq r_{3}$, where $\eta>0$ is chosen so that

$$
\mu \Gamma \eta>1
$$

Thus, if $x \in \partial \Omega_{r_{3}}$, then

$$
f(x(t)) \geq \eta x(t), \quad t \in[0, \omega] .
$$

Thus, Corollary 8 implies that

$$
\left\|T_{\mu} x\right\| \geq \mu \Gamma \eta\|x\|>\|x\| \quad \text { for } x \in \partial \Omega_{r_{3}} .
$$

It follows from Lemma 4 that $T_{\mu}$ has a fixed point $x_{1}(t) \epsilon$ $\bar{\Omega}_{r_{1}} \backslash \Omega_{r_{3}}$ which is a positive periodic solution of (6) for $\mu<\mu_{1}$ satisfying

$$
r_{3}<\left\|x_{1}\right\|<r_{1} \text {. }
$$

On the other hand, since $\lim _{x \rightarrow \infty}(f(x) / x)=\infty$, by Lemma 6, there is $\Delta>0$ such that if $R>\Delta, T_{\mu}$ is defined on $K \backslash \Omega_{R}$ and $g(t) f(x(t))+e(t)$ is nonnegative. Furthermore, $T_{\mu}\left(K \backslash \Omega_{R}\right) \subset K$. For a fixed number $r_{2}>\max \left\{\Delta, r_{1}\right\}$, and Corollary 15 implies that there exists a $0<\mu_{0}<\mu_{1}$ such that we have, for $\mu<\mu_{0}$,

$$
\left\|T_{\mu} x\right\|<\|x\| \quad \text { for } x \in \partial \Omega_{r_{2}} .
$$

Since $\lim _{x \rightarrow \infty}(f(x) / x)=\infty$, there is a positive number $r^{\prime}$ such that

$$
f(x) \geq \eta x
$$

for $x \in \mathbb{R}_{+}$and $x \geq r^{\prime}$, where $\eta>0$ is chosen so that

$$
\mu \Gamma \eta>1
$$

Let $r_{4}=\max \left\{2 r_{2},\left(1 / \sigma_{1}\right) r^{\prime}\right\}>\Delta$. If $x \in \partial \Omega_{r_{4}}$, then

$$
\min _{t \in[0, \omega]} x(t) \geq \sigma_{1}\|x\|=\sigma_{1} r_{4} \geq r^{\prime}
$$

which implies that

$$
f(x(t)) \geq \eta x(t) \quad \text { for } t \in[0, \omega] .
$$

Again, Corollary 8 implies that

$$
\left\|T_{\mu} x\right\| \geq \mu \Gamma \eta\|x\|>\|x\| \quad \text { for } x \in \partial \Omega_{r_{4}} .
$$

It follows from Lemma 4 that $T_{\mu}$ has a fixed point $x_{2}(t) \epsilon$ $\bar{\Omega}_{r_{4}} \backslash \Omega_{r_{2}}$, which is a positive periodic solution of (6) for $\mu<\mu_{0}$ satisfying

$$
r_{2}<\left\|x_{2}\right\|<r_{4}
$$

Noting that

$$
r_{3}<\left\|x_{1}\right\|<r_{1}<r_{2}<\left\|x_{2}\right\|<r_{4},
$$

we can conclude that $x_{1}$ and $x_{2}$ are the desired distinct positive solutions of (6) for $\mu<\mu_{0}$.

(c) Since $\lim _{x \rightarrow 0} f(x)=\infty$, by Lemma 6 , there is a $\delta>0$ such that if $0<r<\delta$, then $T_{\mu}$ is defined and $g(t) f(x(t))+e(t)$ is nonnegative. Now for a fixed number $r_{1}<\delta$, Corollary 15 implies that there exists a $\mu_{1}>0$ such that we have, for $\mu<\mu_{1}$,

$$
\left\|T_{\mu} x\right\|<\|x\| \quad \text { for } x \in \partial \Omega_{r_{1}} .
$$


On the other hand, in view of the assumption $\lim _{x \rightarrow 0} f(x)=\infty$, there is a positive number $0<r_{2}<r_{1}<\delta$ such that

$$
f(x) \geq \eta x
$$

for $x \in \mathbb{R}_{+}$and $0<x \leq r_{2}$, where $\eta>0$ is chosen so that

$$
\mu \Gamma \eta>1 \text {. }
$$

Thus, if $x \in \partial \Omega_{r_{2}}$, then

$$
f(x(t)) \geq \eta x(t), \quad t \in[0, \omega] .
$$

Thus, Corollary 8 implies that

$$
\left\|T_{\mu} x\right\| \geq \mu \Gamma \eta\|x\|>\|x\| \quad \text { for } x \in \partial \Omega_{r_{2}} .
$$

Lemma 4 implies that $T_{\mu}$ has a fixed point $x_{1} \in \bar{\Omega}_{r_{1}} \backslash \Omega_{r_{2}}$. The fixed point $x_{1} \in \bar{\Omega}_{r_{1}} \backslash \Omega_{r_{2}}$ is the desired positive periodic solution of (6).

Case 2. In this case, replacing assumptions (14) and (21) by assumption (32), we can get similar existence results which we omit here.

We illustrate our results with some examples.

Example 18. Consider the following singular equation:

$$
\begin{aligned}
& x^{\prime \prime \prime}(t)+3 x^{\prime \prime}(t)+\frac{41}{20} x^{\prime}(t)+\frac{1}{20} x(t) \\
& =\mu(1+\cos 2 \pi t)\left(\frac{1}{x(t)}+x(t)^{1 / 2}\right)+\mu e^{\sin 2 \pi t},
\end{aligned}
$$

where $\mu$ is a constant and $\mu>0$.

Comparing (100) to $(6)$, we see that $f(x)=(1 / x(t))+$ $x(t)^{1 / 2}, g(t)=1+\cos 2 \pi t, e(t)=e^{\sin 2 \pi t}, \omega=1 a(t)=$ $3, b(t)=41 / 20, c(t)=1 / 20$. Take $p(t)=2, q(t)=1 / 20, \rho=1$; then Case 1 holds. By calculation, we get $R=1 / 40, Q=$ $(1 / 40)\left(1+e^{2}\right)^{2}, A=2, B=1 / 20, \alpha=1+\sqrt{1-(1 / 20)} \approx$ $1.975, \beta=1-\sqrt{1-(1 / 20)} \approx 0.025$, and we have $1 / 40<$ $\left(e^{2}-1\right) /\left(1+e^{2}\right)^{2} \approx 0.0907, A^{2}=4>4 B=1 / 5$; then (14) and (21) hold. Moreover, $g(t) \geq 0, f(t, x)=(1 / x(t))+$ $x(t)^{1 / 2}, \lim _{x \rightarrow 0} f(x)=\infty, \lim _{x \rightarrow \infty}(f(x) / x)=0$; then $\left(H_{1}\right)$ and $\left(H_{2}\right)$ hold. So, by Theorem 16(a), we can get that (100) has positive periodic solution.

Example 19. Consider the following singular equation:

$$
\begin{gathered}
x^{\prime \prime \prime}(t)+2 x^{\prime \prime}(t)+(1+\alpha(t)) x^{\prime}(t)+\left(\alpha(t)+\alpha^{\prime}(t)\right) x(t) \\
=\mu(1+\sin 2 \pi t)\left(\frac{1}{x(t)}+x(t)^{3}\right)+\mu\left(e^{\cos 2 \pi t}-\frac{1}{e}\right),
\end{gathered}
$$

where $\mu$ is a constant and $\mu>0, \alpha(t) \in C^{1}(\mathbb{R})$ is 1-periodic function and $\int_{0}^{1} \alpha(t) d t \neq 0, \alpha: \mathbb{R} \rightarrow \mathbb{R}^{+}$is continuous, and $\alpha_{\infty} \leq(e-1) /(e+1)^{2} \leq 1 / 4$; here, $\alpha_{\infty}=\max _{t \in[0,1]} \alpha(t)$.
Comparing (101) to (6), we see that $f(x)=(1 / x(t))+$ $x(t)^{3}, g(t)=1+\sin 2 \pi t, e(t)=e^{\cos 2 \pi t}-(1 / e), \omega=$ $1 a(t)=2, b(t)=1+\alpha(t), c(t)=\alpha(t)+\alpha^{\prime}(t)$. Take $p(t)=$ $1, q(t)=\alpha(t), \rho=1$; then Case 1 holds. By calculation, we get $R=\int_{t}^{t+1}\left(e^{s-t} /(e-1)\right) \alpha(s) d s, Q=(1+e)^{2} \int_{t}^{t+1}\left(e^{s-t} /(e-\right.$ 1)) $\alpha(s) d s, A=1, B=e^{\int_{0}^{1} \ln \alpha(t) d t}$. From $\alpha_{\infty} \leq(e-1) /(e+1)^{2} \leq$ $1 / 4$, we have $(1 / Q \omega)\left[\exp \left(\int_{0}^{\omega} p(u) d u\right)-1\right]=(e-1) /(1+$ $e)^{2} \int_{t}^{t+1}\left(e^{s-t} /(e-1)\right) \alpha(s) d s \geq(e-1) /(e+1)^{2} \alpha_{\infty} \geq 1$, and $4 B=4 e^{\int_{0}^{1} \ln \alpha(t) d t} \leq 4 \alpha_{\infty} \leq 1=A^{2}$; then (14) and (21) hold. Moreover, $g(t) \geq 0, e(t) \geq 0, f(t, x)=(1 / x(t))+$ $x(t)^{3}, \lim _{x \rightarrow 0} f(x)=\infty, \lim _{x \rightarrow \infty}(f(x) / x)=\infty$; then $\left(H_{1}\right)$ and $\left(H_{2}\right)$ hold. So, by Theorem 16(b), we can get that (100) has two positive periodic solutions.

\section{Conflict of Interests}

The authors declare that there is no conflict of interests regarding the publication of this paper.

\section{Acknowledgment}

Research is supported by NSFC Project (nos. 11326124 and 11271339).

\section{References}

[1] T. R. Ding, "A boundary value problem for the periodic Brillouin focusing system," Acta Scientiarum Naturalium Universitatis Pekinensis, vol. 11, pp. 31-38, 1965.

[2] Z. B. Cheng and J. L. Ren, "Periodic and subharmonic solutions for Duffing equation with a singularity," Discrete and Continuous Dynamical Systems A, vol. 32, no. 5, pp. 1557-1574, 2012.

[3] Z. B. Cheng and J. L. Ren, "Studies on a damped differential equation with repulsive singularity," Mathematical Methods in the Applied Sciences, vol. 36, no. 8, pp. 983-992, 2013.

[4] J. F. Chu, P. J. Torres, and M. R. Zhang, "Periodic solutions of second order non-autonomous singular dynamical systems," Journal of Differential Equations, vol. 239, no. 1, pp. 196-212, 2007.

[5] A. Fonda, R. Manásevich, and F. Zanolin, "Subharmonic solutions for some second-order differential equations with singularities," SIAM Journal on Mathematical Analysis, vol. 24, no. 5, pp. 1294-1311, 1993.

[6] A. Fonda, R. Toader, and F. Zanolin, "Periodic solutions of singular radially symmetric systems with superlinear growth," Annali di Matematica Pura ed Applicata, vol. 191, no. 2, pp. 181204, 2012.

[7] P. Habets and L. Sanchez, "Periodic solutions of some Liénard equations with singularities," Proceedings of the American Mathematical Society, vol. 109, no. 4, pp. 1035-1044, 1990.

[8] X. Li and Z. H. Zhang, "Periodic solutions for damped differential equations with a weak repulsive singularity," Nonlinear Analysis: Theory, Methods \& Applications, vol. 70, no. 6, pp. 2395-2399, 2009.

[9] P. J. Torres, "Non-collision periodic solutions of forced dynamical systems with weak singularities," Discrete and Continuous Dynamical Systems A, vol. 11, no. 2-3, pp. 693-698, 2004. 
[10] P. Torres, "Weak singularities may help periodic solutions to exist," Journal of Differential Equations, vol. 232, no. 1, pp. 277284, 2007.

[11] J. Xia and Z. H. Wang, "Existence and multiplicity of periodic solutions for the Duffing equation with singularity," Proceedings of the Royal Society of Edinburgh A: Mathematics, vol. 137, no. 3, pp. 625-645, 2007.

[12] M. R. Zhang, "Periodic solutions of Liénard equations with singular forces of repulsive type," Journal of Mathematical Analysis and Applications, vol. 203, no. 1, pp. 254-269, 1996.

[13] H. Y. Wang, "Positive periodic solutions of singular systems with a parameter," Journal of Differential Equations, vol. 249, no. 12, pp. 2986-3002, 2010.

[14] J. F. Chu and Z. C. Zhou, "Positive solutions for singular non-linear third-order periodic boundary value problems," Nonlinear Analysis: Theory, Methods \& Applications, vol. 64, no. 7, pp. 1528-1542, 2006.

[15] B. Mehri and M. A. Niksirat, "Some computable results on the existence of periodic solutions for singular non-autonomous third order systems," Applied Mathematics and Computation, vol. 163, no. 1, pp. 51-60, 2005.

[16] Y. X. Li, "Positive periodic solutions for fully third-order ordinary differential equations," Computers \& Mathematics with Applications, vol. 59, no. 11, pp. 3464-3471, 2010.

[17] A. P. Palamides and G. Smyrlis, "Positive solutions to a singular third-order three-point boundary value problem with an indefinitely signed Green's function," Nonlinear Analysis: Theory, Methods \& Applications, vol. 68, no. 7, pp. 2104-2118, 2008.

[18] J. Sun and Y. Liu, "Multiple positive solutions of singular thirdorder periodic boundary value problem," Acta Mathematica Scientia, vol. 25, no. 1, pp. 81-88, 2005.

[19] J. L. Ren, Z. B. Cheng, and Y. L. Chen, "Existence results of periodic solutions for third-order nonlinear singular differential equation," Mathematische Nachrichten, vol. 286, no. 10, pp. 1022-1042, 2013.

[20] Y. Wang, H. Lian, and W. G. Ge, "Periodic solutions for a second order nonlinear functional differential equation," Applied Mathematics Letters, vol. 20, no. 1, pp. 110-115, 2007.

[21] P. Torres, "Existence of one-signed periodic solutions of some second-order differential equations via a Krasnoselskii fixed point theorem," Journal of Differential Equations, vol. 190, no. 2, pp. 643-662, 2003.

[22] M. Krasnoselskii, Positive Solutions of Operator Equation, Noordhoff, Groningen, The Netherlands, 1964.

[23] H. Y. Wang, "On the number of positive solutions of nonlinear systems," Journal of Mathematical Analysis and Applications, vol. 281, no. 1, pp. 287-306, 2003. 


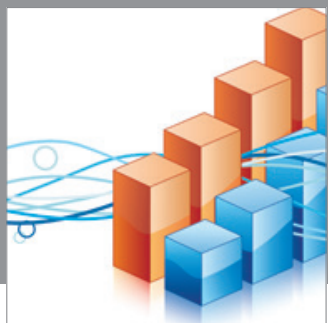

Advances in

Operations Research

mansans

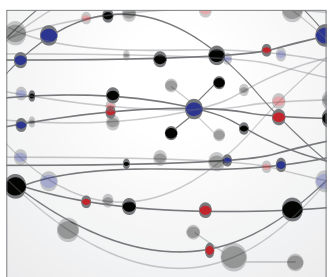

The Scientific World Journal
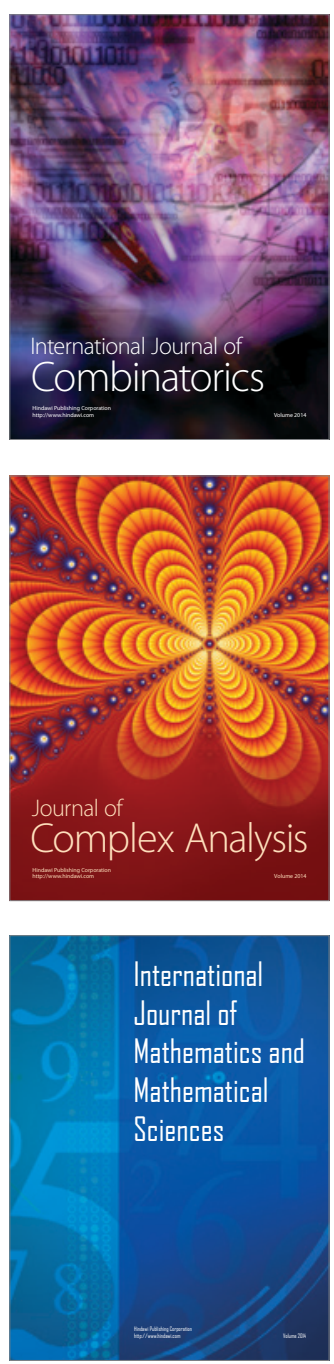
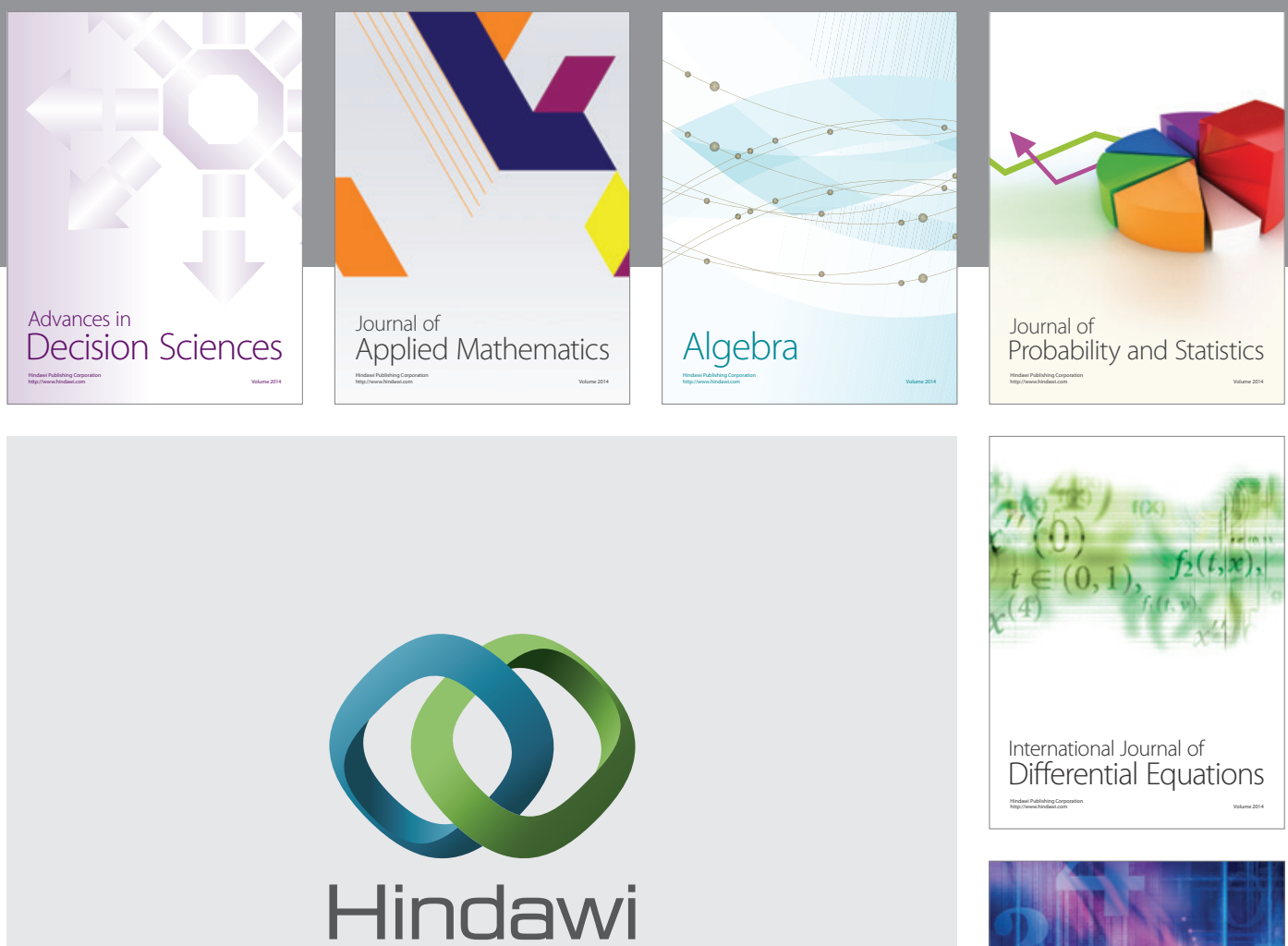

Submit your manuscripts at http://www.hindawi.com
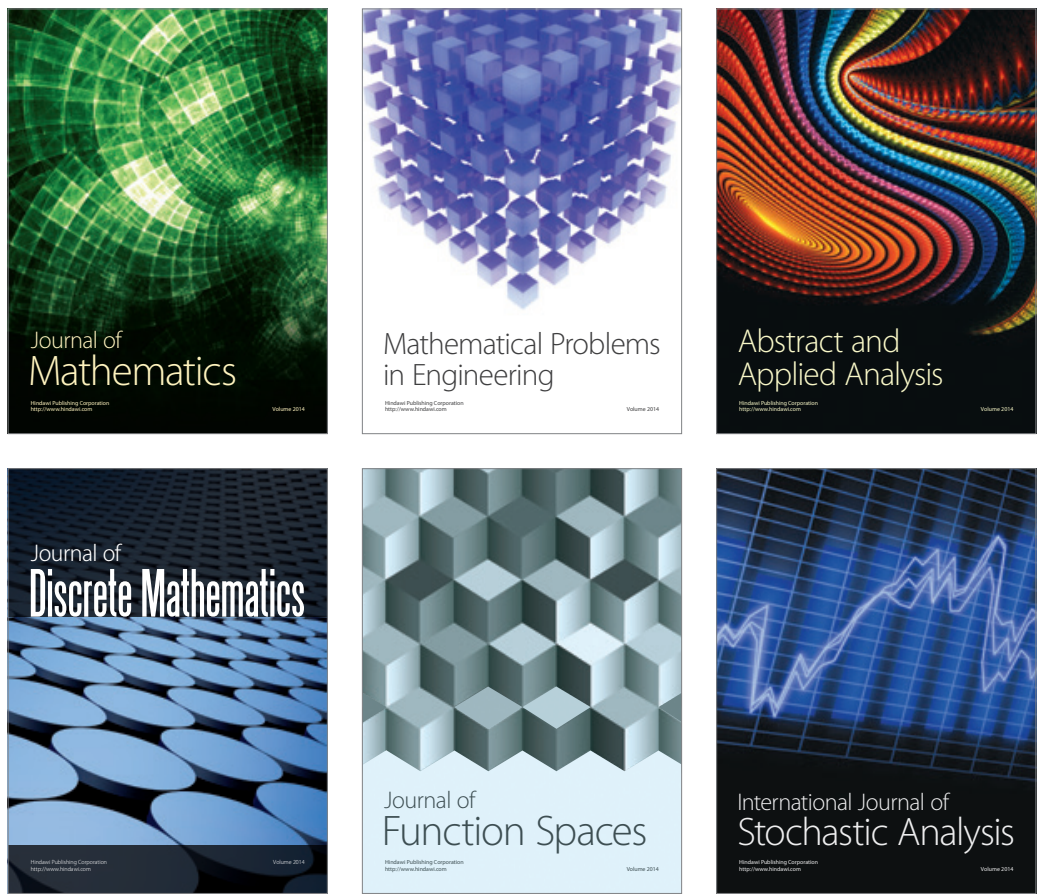

Journal of

Function Spaces

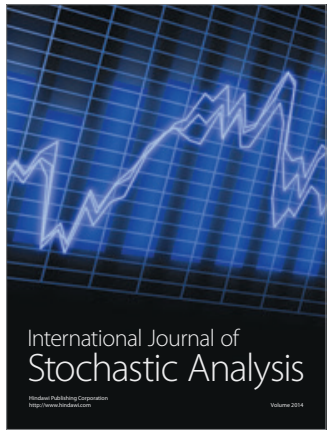

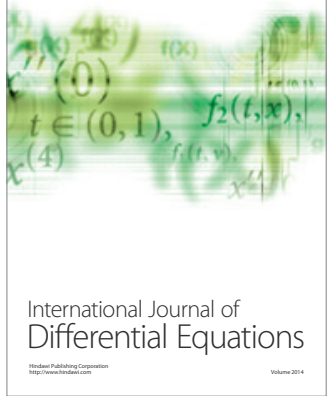
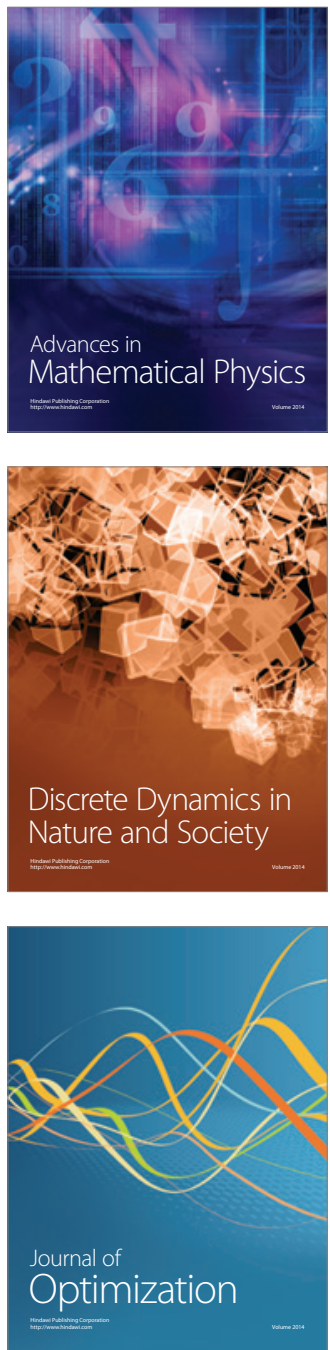